cancer vaccination opportunity when combined in heterologous prime-boost regimen.

Materials and Methods Mice were vaccinated with subcutaneous (s.c.) injection of KISIMA-TAA vaccine and/or with intravenous injection of VSV-GP-TAA in different settings. Immunogenicity was assessed by measuring the peripheral antigen-specific response. Anti-tumoral efficacy as well as in depth monitoring of TILs and tumor microenvironment modulation were assessed following therapeutic vaccination in different tumor models. Additionally, transcriptome and immunohistochemistry analyses of the TC-1 tumor have been performed. Combination of heterologous prime-boost with checkpoint blockade PD-1 therapy has been assessed.

Results Priming with KISIMA-TAA followed by VSV-GP-TAA boost induced a large pool of polyfunctional and persistent antigen-specific cytotoxic $\mathrm{T}$ cells in the periphery as well as within the tumor in several tumor models. Frequencies of antigen specific $\mathrm{T}$ cells are significantly higher than the respective homologous vaccinations. Additionally, transcriptome analysis of a cold tumor model revealed profound changes in the tumor microenvironment upon heterologous vaccination, including a strong upregulation of gene signatures of several pro-inflammatory cytokines and chemokines required for antitumor immunity along with dendritic and $\mathrm{T}$ cell trafficking and activation. This was corroborated by flow-cytometric analysis of tumor-infiltrating leukocytes showing massive $\mathrm{CD}^{+}$ and $\mathrm{CD}^{+}{ }^{+} \mathrm{T}$ cell infiltration as well as repolarization of M2like macrophages towards M1-phenotype. The presence of the $\mathrm{CD}^{+} \mathrm{T}$ cells within the tumor core was confirmed by immunohistochemistry analysis. Moreover, combining heterologous vaccination with checkpoint blockade further improved its therapeutic efficacy and the number of long-term survivors.

Conclusions The KISIMA/VSV-GP heterologous prime-boost approach holds great promise for patients with primary or acquired resistance to checkpoint blockade due to its ability to induce tumor-specific $\mathrm{T}$ cell, improve $\mathrm{T}$ cell infiltration and increase tumor inflammation, even in tumors with limited permissivity for the oncolytic virus.

Disclosure Information E. Belnoue: A. Employment (full or part-time); Significant; AMAL Therapeutics SA. K. Das: None. M. Rossi: A. Employment (full or part-time); Significant; AMAL Therapeutics SA. T. Hofer: None. S. Danklmaier: None. T. Nolden: A. Employment (full or part-time); Significant; Viratherapeutics GmbH. L. Schreiber: None. K. Angerer: None. J. Kimpel: None. S. Hoegler: None. L. Kenner: None. D. von Laer: None. K. Elbers: A. Employment (full or parttime); Significant; Viratherapeutics GmbH. G. Wollmann: None. M. Derouazi: A. Employment (full or part-time); Significant; AMAL Therapeutics SA.

\section{P03.03 NOVEL MACHINE-LEARNING TOOLS IMPROVE COST- EFFECTIVE DEVELOPMENT OF PERSONALISED IMMUNOTHERAPIES: LOWERING FALSE POSITIVE RATES IN THE SEARCH FOR ACTIONABLE IMMUNOGENIC NEOANTIGENS}

\author{
${ }^{1} \mathrm{C}$ Bogaert, 'L Van Oudenhove*, 'L Pfitzer, ${ }^{2} \mathrm{~N}$ Mill, ${ }^{1} \mathrm{~B}$ Fant. ${ }^{1} \mathrm{myN} N \mathrm{O}$, Ghent, Belgium; \\ ${ }^{2}$ University of Zurich, Zurich, Switzerland
}

10.1136/jitc-2021-ITOC8.26

Background Personalised immunotherapy approaches rely on the ability of tumour-derived neoantigens to elicit a T-cell immune reaction able to recognise and kill the tumour cells expressing them. Clinical attempts to leverage the power of neoantigens have however yielded mixed results. This can mostly be attributed to the difficulty of finding truly immunogenic peptides from the set of novel peptides generated by mutations in a given cancer patient. In silico approaches can help alleviate this heavy cost by reducing the neoantigen search space, prioritising epitopes based on various parameters such as epitope expression or MHC binding likelihood. Here we present a suite of tools aimed at further assisting clinicians in selecting the most actionable peptides from a set of potential candidates.

Materials and Methods We developed neoMS, a neural network algorithm able to predict epitope presentation at the cell surface with unparalleled performance. Furthermore, the neoIM algorithm is able to discriminate, in an HLA-agnostic fashion, which of the presented peptides will elicit a T-cell immune reaction. This first-in-class algorithm is a random forest classifier specifically trained to classify short peptides of length 9-11 amino acids as immunogenic or non-immunogenic.

Results The neoMS model achieves up to 0.61 precision at recall 0.4 on its test set, vastly outperforming the current industry standards. In addition, due to his sequence-based comparison method, neoMS exhibits extrapolation capabilities, achieving non-zero predictive power when evaluated on ground truth ligandome data derived from an HLA allele completely absent from the training set. In some cancer indications we showed, moreover, that the neoMS-predicted rate of neoantigen presentation can be used in combination with tumour mutational burden as a high-specificity predictor of response to immune checkpoint inhibitor treatment. neoIM vastly outperforms the currently available methods and can predict peptide immunogenicity with high accuracy (AUC $=0.88$ ). Interestingly, neoIM confirmed ELISPOT data obtained by Dillon et al. (2017) showing a response in 4 out of 11 breast cancer patients to a vaccine consisting of 9 MHC class-I restricted breast cancer-associated peptides. The 2 antigens that resulted in a CD8 + T-cell specific response were predicted by neoIM as the highest scoring showing its potential in finding the truly immunogenic neoantigens.

Conclusions Taken together, these tools decrease false positive rates significantly as they enable improved identification of immunogenic peptides and the predictions correlate with intensity of immune response and clinical benefits. As such, these tools represent a cost-efficient preliminary step in the search for actionable, immunogenic neoantigens.

\section{REFERENCE}

1. Dillon PM, Petroni GR, Smolkin ME, Brenin DR, Chianese-Bullock KA, Smith KT, Olson WC, Fanous IS, Nail CJ, Brenin CM, Hall EH, Slingluff CL Jr. A pilot study of the immunogenicity of a 9-peptide breast cancer vaccine plus poly-ICLC in early stage breast cancer. J Immunother Cancer 2017 Nov 21;5(1):92.

Disclosure Information C. Bogaert: None. L. Van Oudenhove: None. L. Pfitzer: None. N. Mill: None. B. Fant: None.

\section{P03.04 NOVEL ALGORITHM DISCOVERS UP TO 35 PERCENT MORE EPITOPES TRANSLATED FROM NON-CODING REGIONS IN COLD TUMOURS}

C Bogaert, L Van Oudenhove*, B Fant. mNEO, Ghent, Belgium

10.1136/jitc-2021-ITOC8.27 
Background Currently, most neoantigen pipelines often focus on the detection of neoantigens derived from mutations in the coding regions of the genome. However, in some cancer indications, the number of mutations detectable in tumours can be very low (low tumour mutational burden). This limits the number of actionable neoantigens and results in so-called 'cold' tumours. In these cases, non-canonical neoantigens resulting from alterations in non-coding regions of the human genome could represent a high potential alternative for treatment.

Indeed, recent research has revealed that previously presumed non-coding regions of the human genome, such as long non-coding RNAs (lncRNAs), can contain translatable small open reading frames (smORFs) generating micropeptides. Some of these micropeptides have already been shown to be involved in cancer development, but these small peptides could also represent a high potential source of non-canonical neoantigens for personalised therapy.

Materials and Methods Here, we present smORFin, a machine learning algorithm specifically trained to identify smORFs in transcripts and to assess their coding potential. While most tools are focused on longer sequences, smORFin is specifically developed to target small ORFs $(<303$ nucleotides). Furthermore, smORFin also accounts for smORFs with alternative initiation codons, thereby improving its sensitivity for the detection of novel unannotated smORFs.

In addition, the impact of mutations in allegedly non-coding regions of tumour genomes and its influence on the neoantigen repertoire, was evaluated through integration of smORFin in a neoantigen identification pipeline targeting lncRNA-derived mutated epitopes; lncRNeos.

Results The smORFin model reaches a precision of 0.98 and an accuracy of 0.95 on its testing dataset. Using this new prediction tool, a library of human smORFs was assembled, the so-called smORFeome. This library of smORFs, and their associated proteins, was evaluated as a reference for spectrum to peptide matching in mass spectrometry data (MS) analysis. Indeed, the evaluation of seven MS samples revealed and validated the presence of smORFeome-related micropeptides and HLA-I associated epitopes originating from smORFs.

Furthermore, it was observed that IncRNA-derived epitopes only represent a minor fraction of the total neoantigen load. Strikingly, when only focusing on tumours with a low neoantigen load, IncRNeos represented up to $27 \%$ of the total neoantigen load. This indicates that for tumours with a low TMB, and therefore with a low neoantigen load, lncRNeos allows to significantly expand the neoantigen repertoire. Biological in vivo/in vitro validation remains necessary to assess the existence, presentation, and actionability of lncRNeos.

Conclusions A novel random forest-based algorithm was developed to address the need for reliable identification of lncRNA-born smORFs. Furthermore, the integration of this prediction algorithm in a neoantigen pipeline allowed the identification of lncRNA-derived neoantigens and marks them as a potential novel source for personalised immunotherapy.

Disclosure Information C. Bogaert: None. L. Van Oudenhove: None. B. Fant: None.

\section{P03.06 DECIPHERING VACCINE-INDUCED NEOEPITOPE-SPECIFIC T CELL RESPONSES IN A PATIENT WITH H3K27M- MUTANT MIDLINE GLIOMA WHO GRADE 4}

1,2 K Lindner* ${ }^{3} \mathrm{~K}$ Kromer, ${ }^{1} \mathrm{~K}$ Jähne 1,4 ${ }^{3} \mathrm{JM}$ Lindner, ${ }^{1,2,4} \mathrm{M}$ Platten, ${ }^{1,4} \mathrm{~K}$ Sahm. ${ }^{1} \mathrm{DKFZ}$ Heidelberg, Heidelberg, Germany; ${ }^{2}$ National Center for Tumor Diseases (NCT), Heidelberg, Germany; ${ }^{3}$ BioMed X, Heidelberg, Germany; ${ }^{4}$ Department of Neurology, Medical Faculty Mannheim, Mannheim, Germany

\subsection{6/jitc-2021-ITOC8.28}

Background K27M-mutant histone-3 (H3K27M) defines a clinically and molecularly distinct entity of diffuse midline gliomas WHO grade 4 with an unfavorable prognosis. From an immunological perspective, H3K27M constitutes a cancer neoepitope: in a syngeneic MHC-humanized mouse model, an H3K27M-specific long peptide vaccine induced mutation-specific $\mathrm{T}$ cells responses capable of inhibiting growth of H3K27M-expressing tumors.

Materials and Methods Here, we describe clinical response of a patient diagnosed with H3K27M-mutant midline gliomas to H3K27M-specific peptide vaccination and exploit vaccineinduced $\mathrm{T}$ cell phenotypes.

Results Repeated peptide vaccinations were well tolerated and resulted in long-term response after pseudoprogression. Longitudinal immune monitoring showed induction of $\mathrm{H} 3 \mathrm{~K} 27 \mathrm{M}$ specific CD4-driven $\mathrm{T}$ cell responses in the peripheral blood. Within the cerebrospinal fluid, expansion of HLA-specific vaccine-induced $\mathrm{T}$ cell receptor (TCR) clones was observed and associated with distinct HLA types.

Conclusions Identification of vaccine-induced TCR clones within the peripheral blood and CSF of patients with H3K27M-mutant midline glioma may be used to prioritize TCRs for adoptive $\mathrm{T}$ cell therapy.

Disclosure Information K. Lindner: None. K. Kromer: None. K. Jähne: None. L. Bunse: E. Ownership Interest (stock, stock options, patent or other intellectual property); Modest; Patent: US20180155403A1 (Histone Anti-Cancer Vaccines). C. Tan: None. I. Poschke: None. E. Green: None. J.M. Lindner: None. M. Platten: C. Other Research Support (supplies, equipment, receipt of drugs or other in-kind support); Modest; Roche. E. Ownership Interest (stock, stock options, patent or other intellectual property); Modest; Patent: US20180155403A1 (Histone Anti-Cancer Vaccines). K. Sahm: E. Ownership Interest (stock, stock options, patent or other intellectual property); Modest; Patent: US20180155403A1 (Histone Anti-Cancer Vaccines).

\section{P03.07 ANALYSIS OF SCRNASEQ FROM THE HUMAN THYMUS NOMINATES GENES POTENTIALLY MISSING FROM CENTRAL TOLERANCE OF CYTOTOXIC T CELLS}

L Blumenberg*, G Atwal, A Dhanik. Regeneron Pharmaceuticals, Tarrytown, NY, USA

\subsection{6/jitc-2021-ITOC8.29}

Background During thymic development, cytotoxic T cells that can bind to and attack self antigens undergo negative selection thus preventing damage to the self tissues. The sparse medullar thymic epithelial cells (mTECs) present in the thymus are responsible for presenting self antigens to $\mathrm{T}$ cells so that they 